\title{
REVIEWS
}

\section{Cholera transmission: the host, pathogen and bacteriophage dynamic}

\author{
Eric J. Nelson*, Jason B. Harris ${ }^{\ddagger}$, J. Glenn Morris Jrll, Stephen B. Calderwood ${ }^{\ddagger}$ \\ and Andrew Camilli*
}

\begin{abstract}
Zimbabwe offers the most recent example of the tragedy that befalls a country and its people when cholera strikes. The 2008-2009 outbreak rapidly spread across every province and brought rates of mortality similar to those witnessed as a consequence of cholera infections a hundred years ago. In this Review we highlight the advances that will help to unravel how interactions between the host, the bacterial pathogen and the lytic bacteriophage might propel and quench cholera outbreaks in endemic settings and in emergent epidemic regions such as Zimbabwe.
\end{abstract}

\section{$\mathrm{O}$ antigen}

The outermost, repeating oligosaccharide portion of LPS, which makes up the outer leaflet of the outer membrane of Gram-negative bacteria.

Cholera toxin A protein toxin produced by $\checkmark$. cholerae that triggers fluid and electrolyte secretion by intestinal epithelial cells
* Howard Hughes Medical Institute and the Department of Molecular Biology and Microbiology, Tufts University School of Medicine, Boston, Massachusetts 02111, USA. ${ }^{\ddagger}$ Division of Infectious Diseases, Massachusetts General Hospital, Boston, Massachusetts 02114, USA. §arvard Medical School, Boston, Massachusetts 02115, USA. Emerging Pathogens Institute, University of Florida, Gainesville, Florida 32611, USA. Correspondence to A.C. e-mail: Andrew.camilli@tufts. edu

doi: $10.1038 /$ nrmicro2204
Diarrhoeal diseases, including cholera, are the leading cause of morbidity and the second most common cause of death among children under 5 years of age globally ${ }^{1,2}$. It is difficult to gauge the exact morbidity and mortality of cholera because the surveillance systems in many developing countries are rudimentary, and many countries are hesitant to report cholera cases to the WHO because of the potential negative economic impact of the disease on trade and tourism. Today, the true burden of cholera is estimated to reach several million cases per year, predominantly in Asia and Africa ${ }^{3}$. With optimal delivery, oral rehydration therapy can lower case fatality rates from the $>20 \%$ seen historically ${ }^{4-6}$ to $<1 \%{ }^{7}$. Much work remains to be done, as 27 countries reported case fatality rates above the $1 \%$ threshold in 2007 (REF. 8).

The causative agent of cholera, the Gram-negative bacterium Vibrio cholerae, is a facultative pathogen that has both human and environmental stages in its life cycle ${ }^{9,10}$. V. cholerae is differentiated serologically on the basis of the $O$ antigen of its lipopolysaccharide (LPS) (FIG. 1). Cholera toxin-producing (toxigenic) strains of the $\mathrm{O} 1$ and $\mathrm{O} 139$ serogroups cause the vast majority of the disease. The $\mathrm{O} 1$ serogroup is subdivided into two phenotypically distinct biotypes, El Tor and classical, the second of which is associated with earlier pandemics. Both biotypes can be further subdivided into two serotypes, Inaba and Ogawa ${ }^{7}$. In the past 20 years, El Tor has replaced the classical biotype ${ }^{11}$; however, the legacy of the classical biotype lingers, as El Tor strains harbouring classical cholera toxin have emerged ${ }^{12-14}$. The 0139 serogroup first appeared in 1992, as a result of a multigene substitution in the $\mathrm{O}$ antigen-coding region of a progenitor O1 El Tor strain ${ }^{15}$. Although the O139 serogroup caused devastating outbreaks in the 1990s, the El Tor strain remains the dominant strain globally ${ }^{11,16,17}$.

An extensive body of literature describes the pathophysiology of cholera. In brief, pathogenic strains harbour key virulence factors that include cholera toxin ${ }^{18}$ and toxin co-regulated pilus (TCP) $)^{19,20}$, a self-binding pilus that tethers bacterial cells together ${ }^{21}$, possibly to resist shearing forces in the host small intestine. Cholera toxin is a secreted $\mathrm{AB}_{5}$-subunit toxin. The $\mathrm{B}$ subunit pentamer binds monosialotetrahexosylgangliosides on absorptive epithelial cells, triggering endocytosis of the enzymatic A subunit, whereupon it ADP ribosylates a subunit of the $\mathrm{G}$ protein that controls adenylyl cyclase activity. Although virulence is multifactorial, cholera toxin is the key factor responsible for the profuse secretory diarrhoea that occurs in infected individuals. Transmissible elements, such as the lysogenic bacteriophage that carries the genes for cholera toxin ${ }^{22}$ and the SXT element that harbours antibiotic resistance genes ${ }^{23}$, will continue to shape the evolution of $V$. cholerae.

The biological and environmental factors that contribute to the dynamics of cholera outbreaks continue to be the subject of intense study. Several review articles covering the importance of environmental drivers in promoting cholera outbreaks have been published ${ }^{24-26}$. In this Review, we focus rather on three biological factors that are thought to have important roles in leading to and shaping cholera outbreaks: host susceptibility, the virulence of $V$. cholerae and lytic bacteriophages. Below, we discuss this trinity of factors as they pertain to the dynamic nature of cholera outbreaks. 


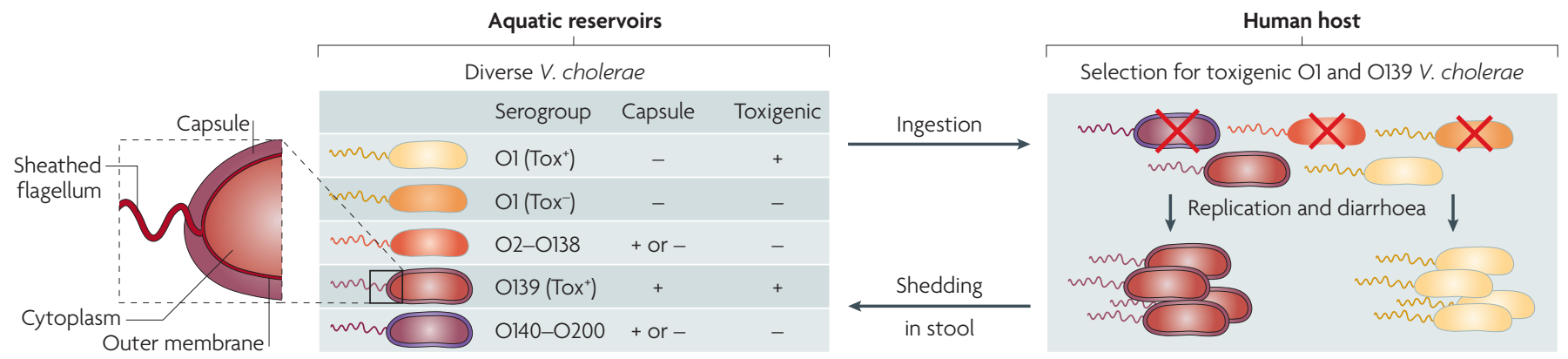

Figure 1 | Phylogenetic relationship of Vibrio cholerae strains. On the basis of the antigenicity of the $\mathrm{O}$ antigen component of the outer membrane lipopolysaccharide, more than 200 serogroups (O1-O200) of Vibrio cholerae exist in aquatic environments. Only a subset of $\mathrm{O} 1$ and $\mathrm{O} 139$ serogroup strains are toxigenic (Tox ${ }^{+}$) and therefore capable of causing cholera when ingested; such strains are selected for in the host. Other strains are non-toxigenic (Tox ${ }^{-}$) and are selected against. Different $\mathrm{O}$ antigen types are indicated by the colour of the outer membrane and sheathed flagellum (the periplasmic space and the inner membrane are not shown). Capsules are present in a subset of strains. Different strain genotypes are indicated by the colour of the cytoplasm; note that Tox ${ }^{+} \mathrm{O} 1$ and $\mathrm{O} 139$ have essentially the same genotype, with the exception of the $\mathrm{O}$ antigen genes.

\section{Toxigenic}

Describing a strain that harbours the genes for toxin production; in the case of V. cholerae, these are the genes for cholera toxin.

\section{Serogroup}

A group of strains sharing the same dominant antigen(s); in the case of $V$. cholerae, this is the lipopolysaccharide $O$ antigen.

\section{Biotype}

A group of strains that share

the same genotype.

Lysogenic

Describing a bacterial strain that harbours a bacteriophage genome within its genome.

Rice water stool Secretory diarrhoea that has the appearance of water that rice has been cooked in.

Vibriocidal antibody An antibody that opsonizes $V$. cholerae sufficiently enough to result in bacterial killing by serum complement

components.

\section{The human host}

Clinical spectrum of $\mathrm{V}$. cholerae infection. Infection with $V$. cholerae produces a clinical spectrum that ranges from asymptomatic colonization to cholera gravis, the most severe form of the disease (TABLE 1). Following host ingestion of contaminated food or water, $V$. cholerae colonizes the small intestine for 12 to 72 hours before symptoms appear. Cholera often begins with stomach cramps and vomiting followed by diarrhoea, which may progress to fluid losses of up to 1 litre per hour ${ }^{27}$. These losses result in severe fluid volume depletion and metabolic acidosis, which may lead to circulatory collapse and death ${ }^{7}$. Rice water stool typically harbours between $10^{10}$ and $10^{12}$ vibrios per litre. Symptomatic patients may shed vibrios before the onset of illness ${ }^{28,29}$ and will continue to shed organisms for 1 to 2 weeks ${ }^{30,31}$. Asymptomatic patients typically shed vibrios in their stool for only 1 day, at approximately $10^{3}$ vibrios per gram of stool ${ }^{32}$. Therefore, the distribution of symptomatic patients influences the quantity of V. cholerae that is shed for subsequent transmission.

The number of symptomatic cases varies by age and by the endemic nature of the disease. In an endemic setting, such as the Ganges River Delta, children are more likely to be hospitalized with severe illness ${ }^{33}$. Over the past 20 years, the preponderance of severe cases has been shifting to younger children, with a peak of severe cases at the age of 2 years ${ }^{34}$. By contrast, in epidemic patterns of transmission, such as when $V$. cholerae is introduced into an immunologically naive population, all age groups seem equally susceptible to symptomatic infection ${ }^{16,35-37}$.

Asymptomatic cases might also contribute to the spread of the organism, albeit at much lower levels than symptomatic patients, and may reflect an important component of the acquired immunity that is seen in some communities. However, asymptomatic cases are often difficult to document. A fourfold rise in the serum vibriocidal antibody titre is a useful measure to identify asymptomatic individuals who may be infected but from whom it is not possible to isolate the organism. Using a positive rectalswab culture or vibriocidal antibody response to define infection, it was recently found that Bangladeshi children under the age of 5 years were 2-3 times as likely to become symptomatic as those over the age of 5 years ${ }^{35}$. The symptom rate across all age brackets in this study was $57 \%^{35}$. This parallels the rates of symptomatic infections that were found in association with classical strains in older literature $\mathrm{e}^{32,38-40}$ but is well above the rates reported for El Tor infections in the $1970 \mathrm{~s}^{40}$. Therefore, asymptomatic cases may still represent roughly half of all cases. Future research on the immunology of asymptomatic patients will help to gauge their contribution to protective immunity at the population level.

Susceptibility to cholera. Host genetic and nutritional factors affect susceptibility to cholera. The ABH histo-blood group antigens are a set of cellular and secreted glycolipids and glycoproteins that are key determinants of host susceptibility to a number of gastrointestinal pathogens; they seem to affect host cell receptor specificity for pathogen and toxin binding. The $\mathrm{O}$ phenotype corresponds to an unmodified $\mathrm{H}$ antigen and is associated with a decreased risk of infection with $V$. cholerae. However, once the host is infected the $\mathrm{O}$ phenotype is associated with an increased risk of severe symptoms; the mechanism for this remains unknown. The prevalence of the O phenotype varies among human populations; its low prevalence in the Ganges River Delta suggests that there is selection against this phenotype in a cholera-endemic area ${ }^{35,41,42}$. In populations with a high prevalence of blood group $\mathrm{O}$, such as those in Latin America, the disease is more severe and requirements for rehydration and hospitalization of infected individuals are substantially higher ${ }^{43,44}$.

Although the $\mathrm{H}$ blood group antigen is the only long recognized genetic factor associated with susceptibility to cholera, other genetic polymorphisms are likely to have been selected for or against, given the historically high mortality rates of cholera. For example, a recent study associated severe cholera with a variant in LPLUNC1 (long palate, lung and nasal epithelium carcinoma-associated protein 1$)^{45}$, a conserved innate immunity protein. The 


\begin{tabular}{llll}
\hline \multicolumn{2}{l}{ Table 1 Clinical spectrum of Vibrio cholerae } & infection \\
& Asymptomatic infection & Mild infection & Severe infection \\
\hline $\begin{array}{l}\text { Symptoms } \\
\text { Dehydration }\end{array}$ & None & $\begin{array}{l}\text { Diarrhoea* } \\
\text { None to mild }\end{array}$ & $\begin{array}{l}\text { Vomiting and profuse diarrhoea } \\
\text { Moderate to severe (hypovolaemic } \\
\text { shock) }\end{array}$ \\
\hline $\begin{array}{l}\text { Stool } \\
\text { characteristics }\end{array}$ & Normal & Loose or watery & Rice water \\
\hline $\begin{array}{l}\text { Vibrios per gram } \\
\text { of stool }\end{array}$ & Up to $10^{5}$ & Up to $10^{8}$ & $10^{7}$ to $10^{9}$ in stool (and vomitus) \\
\hline $\begin{array}{l}\text { Treatment } \\
\text { Mortality }\end{array}$ & None & $\begin{array}{l}\text { Oral rehydration solution } \\
\text { (ORS) }\end{array}$ & $\begin{array}{l}\text { ORS, intravenous fluids and } \\
\text { antibiotics }\end{array}$ \\
\hline None & None & $\begin{array}{l}\text { Untreated: up to } 50 \% \\
\text { Treated: less than } 1 \%\end{array}$
\end{tabular}

*Mild symptoms of Vibrio cholerae infection are indistinguishable from those of numerous other infectious causes of gastroenteritis. ${ }^{\ddagger}$ Antibiotics shorten the duration of symptoms and lessen the total fluid requirement but are not strictly necessary.

expression of LPLUNC1 is upregulated in the small bowel epithelium during acute cholera and may influence the course of $V$. cholerae infection ${ }^{46}$.

Malnutrition, as measured by stunted linear growth, does not seem to be a risk factor for V. cholerae infection ${ }^{35}$. However, deficiency of retinol (vitamin A), a micronutrient that contributes to mucosal immunity, is a risk factor associated with both $V$. cholerae infection and the development of symptomatic illness ${ }^{35}$. Zinc, another micronutrient that contributes to mucosal immunity, can become depleted during diarrhoeal illness ${ }^{47}$. Oral zinc reverses this deficiency in children, resulting in a substantial reduction in stool volume and time to cessation of diarrhoea ${ }^{44}$.

Protective immunity. Several studies demonstrate that clinically apparent $V$. cholerae infection induces protective immunity against subsequent infection (BOX 1). Infection of North American volunteers with the classical and El Tor biotypes provided $100 \%$ and $90 \%$ protection, respectively, from subsequent challenge ${ }^{31,48}$. Similarly, in an endemic setting it was found that greater than $90 \%$ of patients with severe disease due to infection by the classical biotype were protected from future infection, on the basis of the observed versus expected rates of hospitalization for a second episode of cholera in that population ${ }^{33}$; similar results were found in a separate study ${ }^{49}$. The mechanism behind this protective immunity to $V$. cholerae infection and disease is not known.

Several correlates of immunity have been identified. The best characterized serological marker of immunity is the serum vibriocidal antibody titre. This antibody is a complement-dependent bactericidal antibody, the titre of which increases markedly after disease but declines to baseline after 6-9 months ${ }^{7}$. In Bangladesh, vibriocidal antibodies are detectable in most individuals by the age of 10-15 years and are associated with a decreased risk of infection. As V. cholerae is a non-invasive pathogen, these serum antibodies may not contribute directly to protection at the level of the intestinal mucosa but they may be useful in estimating the degree of protective immunity at the population level. Immunoglobulin A antibodies specific to TCP, LPS or the B subunit of cholera toxin correlate with protection from subsequent infection and disease $\mathrm{e}^{35}$.
Vaccine field trials provide a controlled assessment of the durability of adaptive immune responses to $V$. cholerae. In the largest controlled trial, 62,285 participants in Bangladesh were randomized to receive 3 oral doses (6 weeks apart) of a combination of killed whole cells (El Tor and classical) plus the B subunit of cholera toxin (B-WC vaccine), the whole-cell component without the B subunit (WC vaccine) or killed Escherichia coli $\mathrm{K} 12$ as a control. At 3 years of followup, the two vaccines (B-WC and WC) had $50 \%$ and $52 \%$ protective efficacy, respectively ${ }^{50}$. For children $<5$ years of age the efficacy was $23-26 \%$. At 6 months of follow-up, the B-WC vaccine gave $85 \%$ protection $^{51}$. There is debate over whether these data demonstrate an efficacy that is sufficient for large-scale dissemination. New vaccine efforts are underway to produce higher and more durable responses in recipients as well as to lower production costs in order to overcome both the immunological and the production hurdles that limit the feasibility of current cholera vaccines ${ }^{52-55}$.

The part that herd immunity plays in slowing cholera transmission has recently become appreciated. Vaccine efficacy trials randomize individual patients to ensure that protective efficacy reflects only the direct effect of the vaccine $e^{56}$. This means that the licensure of vaccines does not take into account their benefits at the level of the population. The B-WC vaccine trial discussed above had coverage rates that ranged from $4 \%$ to $65 \%$ across different regions ${ }^{50,57}$. When the incidence rates of cholera were compared with the rates of vaccine coverage in specific regions, it was found that the incidence among placebo recipients was inversely correlated with the level of vaccine coverage ${ }^{57}$ : where coverage was high, even those that did not receive the vaccine were still one quarter as likely to get cholera as unvaccinated individuals in areas of low coverage. When herd immunity is incorporated into simulation models of vaccine efficacy, a B-WC vaccination coverage of $50 \%$ in endemic areas yields a $93 \%$ reduction in overall cases ${ }^{58}$. Taken together, these studies argue that the B-WC vaccine may provide sufficient protection for large-scale vaccination in endemic regions and that herd immunity is likely to play an important part in limiting the transmission of cholera. 


\section{Box 1 | The human immune response to Vibrio cholerae}

Innate response

Cholera is thought to be a prototypical non-inflammatory

infection. There are often no gross changes to the

intestinal mucosa or the architectural integrity of

the small bowel. However, there is upregulation

of pro-inflammatory cytokines (including interleukin-1 $\beta$

and tumour necrosis factor) the expression of diverse

bactericidal proteins and migration of neutrophils to the

lamina propria during acute cholera. Natural variability in

the innate immune response may affect susceptibility,

suggested by the finding that a polymorphism in the

promoter region of the LPLUNC1 (long palate, lung and

nasal epithelium carcinoma-associated protein 1) gene

is associated with increased risk of cholera.

\section{Adaptive response}

How the adaptive immune response to cholera mediates protection against subsequent disease is unknown. As Vibrio cholerae is non-invasive, it has been suggested that intestinal secretory immunoglobulin A (slgA) protects against colonization of the mucosa. Approximately 8 days after the onset of cholera, there is a peak in circulating V. cholerae antigen-specific lymphocytes that express gut-homing chemokine-receptors (see the figure) ${ }^{112}$. These lymphocytes soon become undetectable in the blood, as they return to the intestinal mucosa, where they lead to a rise in intestinal slgA secretion. Serum antibody responses such as the vibriocidal antibody response also peak 1-3 weeks after infection. Although high serum titres of vibriocidal antibody and cholera toxin-specific $\lg A$ are correlated with protection against infection, these antibodies decrease to baseline levels by a year after infection, long before protective immunity to cholera wanes. Similarly, in V. cholerae-infected volunteers, mucosal slgA levels decrease to baseline levels in months. However, when volunteers who no longer have detectable antibodies are re-challenged with $V$. cholerae antigens, they demonstrate a persistent ability to mount an anamnestic immune response, developing peak intestinal antibody secretion as rapidly as within three days. Therefore it is possible that the rapidity of the anamnestic response on re-exposure, rather than preformed antibodies, may mediate protection against cholera. This is supported by recent evidence that cholera induces a memory B cell response that is detectable for at least 1 year after cholera infection ${ }^{113}$.

Transmission from the perspective of the host. Several groups have generated mathematical models using both host and pathogen perspectives to help explain and predict the nature of cholera outbreaks. The goal of most of these models is to accurately reflect the steep rise and fall of cholera cases that is seen biannually in regions around the Bay of Bengal. A model was constructed to incorporate transmission rates, a factor for seasonal variation and a calculation for the number of susceptible individuals on the basis of varying protective immunity from past infection ${ }^{59-61}$. These ideas were built on by testing the hypotheses that a high asymptomatic to symptomatic case ratio is associated with outbreak resolution and that a short interval of protective immunity (2-12 weeks) after infection permits the subsequent outbreaks that are observed in Bangladesh ${ }^{62}$. The results suggest that the combination of many asymptomatic cases and shortlived immunity, rather than silent shedders, produces a model that best reflects epidemiological data from West Bengal. The model has limitations: it assumes an asymptomatic to symptomatic case ratio that is much higher than those observed in recent years and it was tested using mortality rates that are attributable to clinically defined cholera from West Bengal between 1891 and 1940 - an era before the discovery of the rotavirus and enterotoxigenic $E$. coli, which are confounding factors. Despite their drawbacks, these models advance our understanding of the complex dynamics of outbreaks and show how powerful the host response can be in limiting transmission. New models for cholera transmission should continue to include factors for seasonal variation, the ratio of asymptomatic to symptomatic cases and decay rates of protective immunity.

\section{The pathogen}

Infectious doses in animal models. Infecting the human host is a multistage process: $V$. cholerae must be ingested at a dose that is sufficient to overcome innate immune defences, then express virulence factors to colonize the small intestine and, finally, coordinate an exit from the host to facilitate transmission ${ }^{63}$ (FIG. 2). Animal models of cholera are largely measures of the success of the first two stages ${ }^{64}$. Using the infant mouse model of infection, it was shown that $V$. cholerae that is shed in human rice water stool are in a hyperinfectious state, exhibiting an $\mathrm{ID}_{50}$ of 10-100 cells compared with approximately 500 cells for $V$. cholerae grown in vitro ${ }^{65-67}$. Hyperinfectivity has also been documented in Citrobacter rodentium ${ }^{68}$, and hyperinfectious $V$. cholerae can be reproduced with passage through a mouse model of infection ${ }^{68,69}$. The definition and phenotype of hyperinfectivity is controversial because of complications in choosing the most relevant culture conditions for the growth of the control strain.

The molecular mechanisms that contribute to hyperinfectivity in V. cholerae are multifactorial. Microarray studies have shown that the global transcriptional profile in hyperinfectious $V$. cholerae from rice water stool is different from that of $V$. cholerae grown in vitro or upper small bowel $V$. cholerae expelled in vomitus ${ }^{65,70,71}$ (FIG. 3). The majority of the known virulence genes, including those 


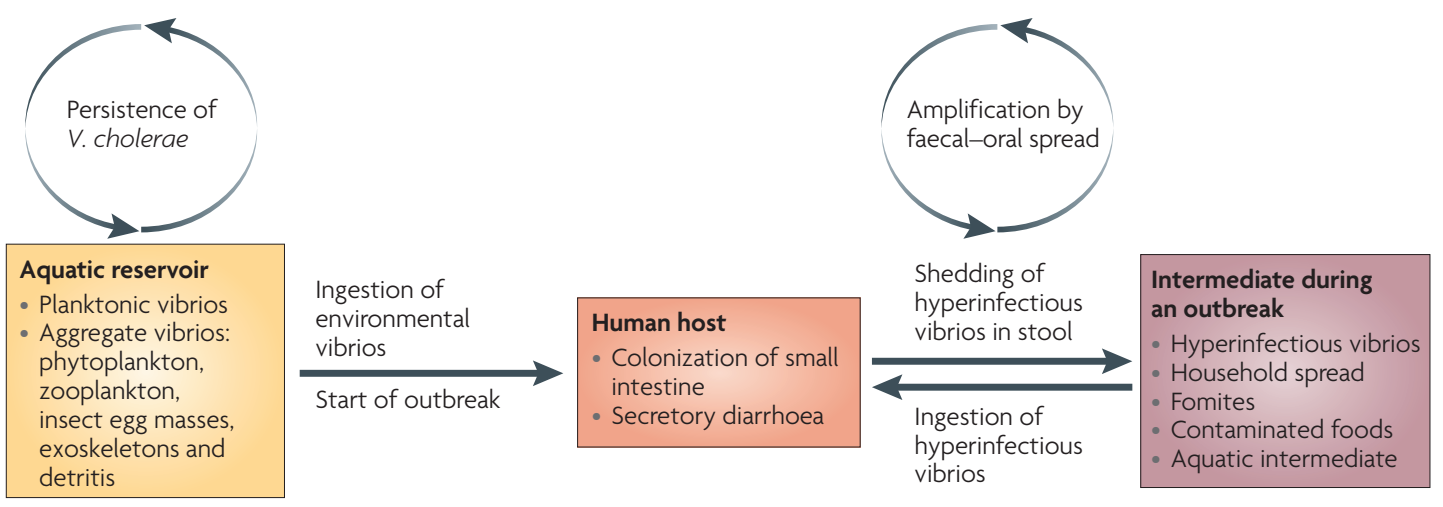

Figure 2 | Life cycle of pathogenic Vibrio cholerae. Toxigenic strains of Vibrio cholerae persist in aquatic environments alongside non-toxigenic strains, aided by biofilm formation on biological surfaces and use of chitin as a carbon and nitrogen source. On ingestion of these aquatic-environment-adapted bacteria in contaminated food or water, toxigenic strains colonize the small intestine, multiply, secrete cholera toxin and are shed back into the environment by the host in secretory diarrhoea. The stool-shed pathogens are in a transient hyperinfectious state that serves to amplify the outbreak through transmission to subsequent hosts.

for cholera toxin and TCP, are downregulated in rice water stool, but the mechanism for this downregulation remains unknown. Another unique signature is the downregulation of chemotaxis genes, which is surprising given that the planktonic vibrios shed by patients are flagellated and highly motile. It has been shown that this downregulation of chemotaxis is one component of hyperinfectivity, because motile but non-chemotaxing $V$. cholerae mutants are hyperinfectious ${ }^{66,72,73}$.

The transcriptome of $V$. cholerae that is passaged from animals is also unique and has been highlighted by two recent studies. First, there is a coordinated 'escape response' that allows the organism to detach from the intestinal villus in preparation for exit from the host; this is regulated by the stress and stationary phase RNA polymerase sigma factor $\underline{\mathrm{RpoS}}^{74}$. Second, during the later stages of animal infection, $V$. cholerae upregulates the expression of genes that are not required for infection but that are important for survival on passage into the aquatic environment ${ }^{75}$. This pre-induction of environmental survival genes late in the infection may prepare the organism for the harsh selective pressures in pond water, therefore facilitating transmission ${ }^{24,65}$. In most of the studies described above, the bacterial cells that constituted the infectious dose were planktonic cells, not aggregates. There has been one volunteer study in which $V$. cholerae in a rugose form (that is, aggregative and exopolysaccharide-producing) was administered to volunteers; the infectious dose was similar to that seen with planktonic forms, and rugose forms were excreted by the volunteers ${ }^{76}$. In addition, humans shed $V$. cholerae in complex, biofilm-like aggregates ${ }^{67,77,78}$. The $\mathrm{ID}_{50}$ of $V$. cholerae shed in aggregates and the part that these aggregates play in transmission remain to be determined.

Infectious dose in humans. The infectious dose of $V$. cholerae in humans varies greatly depending on the bacterial strain and the host. Doses of $10^{8}-10^{11}$ cells were required to produce consistent colonization in healthy North American volunteers ${ }^{7,29,31}$. The infectious dose drops to $10^{4}-10^{8}$ when a bicarbonate buffer is used to neutralize stomach acid shortly before inoculation; this method yields infection rates of $90 \% \%^{7,29,31}$. In the context of households, food may act as an acid buffer; in volunteer studies, administration of the bacterium as part of a 'meal' of rice, fish, custard and skimmed milk gave results that are comparable to those seen with concurrent administration of bacterium and bicarbonate ${ }^{79}$. In an endemic setting, the infectious dose is unknown. Enumerating V. cholerae in household and environmental samples is historically difficult, demanding a rapid response and the use of fluorescence microscopy to count those bacteria that may have become difficult to culture ${ }^{80}$.

The clinical observation that buffering stomach acid lowers the infectious dose suggests that bacterial genes involved in acid resistance might contribute to virulence. Signature-tagged mutagenesis (STM) was used to find a subset of virulence genes that contribute to acid resistance in vitro ${ }^{81}$. Deleting these genes attenuated $V$. cholerae in the infant mouse model of infection, confirming their role in virulence. Furthermore, exposing wild-type $V$. cholerae to acid just before inoculating the mice produced a large competitive advantage over non-acid-adapted bacteria ${ }^{82}$. These data, along with others, demonstrate that bacteria grown in vitro can be induced to be more infectious through stress application. In addition, these findings may have clinical relevance in areas of the world where reduced stomach acid production (achlorhydria) secondary to Helicobacter pylori infection is prevalent, such as Bangladesh ${ }^{7}$.

Transmission from the perspective of the pathogen. The part that hyperinfectivity plays in the rapid spread of cholera throughout a population at the onset of an outbreak remains untested. $V$. cholerae remains hyperinfectious for at least 5 hours after passage from patients into the aquatic environment, suggesting that hyperinfectivity has a particular role in transmission in areas of high crowding, where it is likely that another person will come into contact with the organism in a relatively short time frame ${ }^{65,70}$. One model for cholera transmission suggests that an 


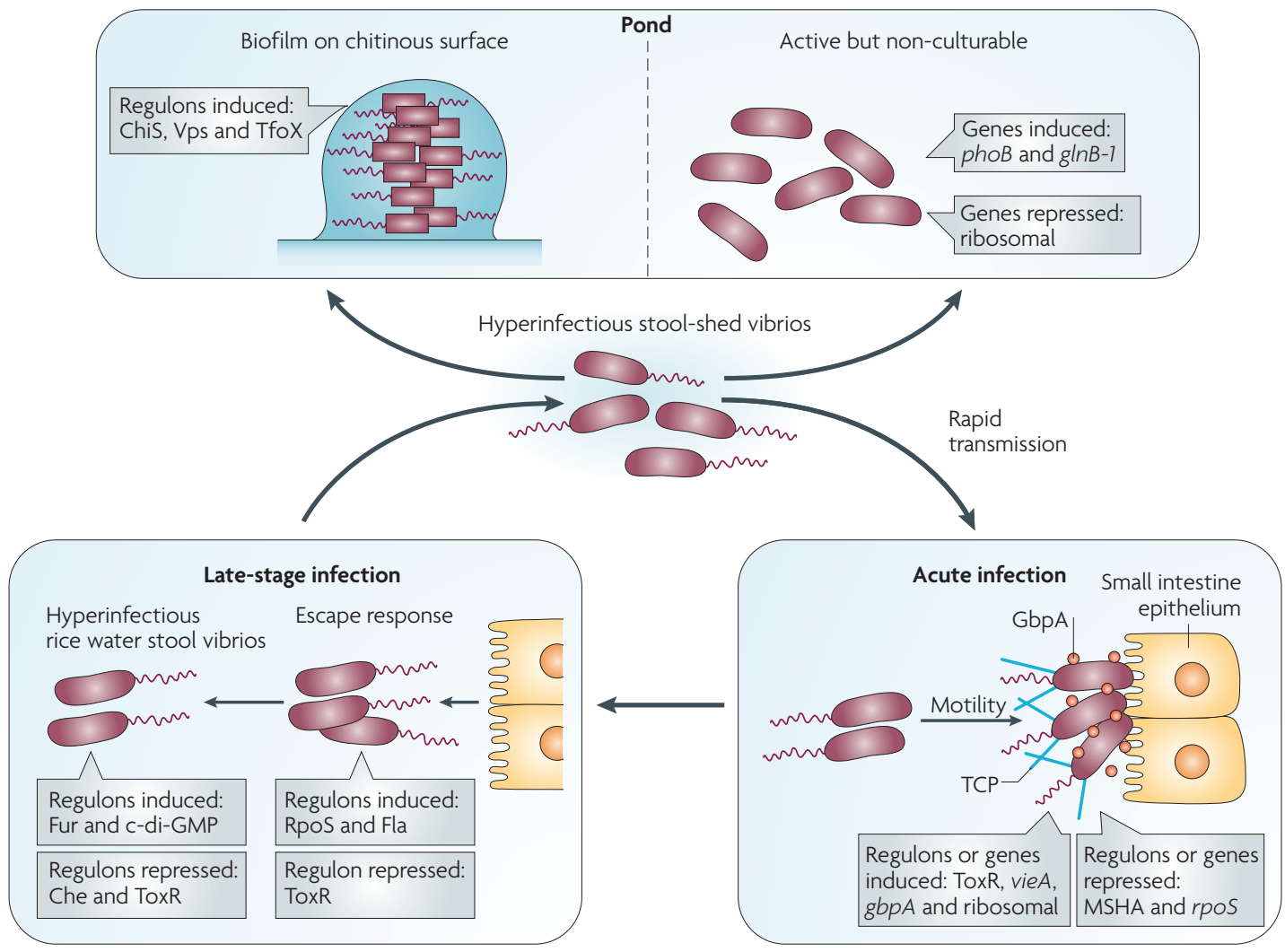

Figure 3 | Vibrio cholerae gene expression patterns at different stages of the life cycle. On ingestion (bottom right panel), Vibrio cholerae uses motility and mucinases to penetrate the mucus gel and $\mathrm{N}$-acetylglucosamine-binding protein $\mathrm{A}$ $(\mathrm{GbpA})$ and other factors to colonize the small intestine epithelium. Substantial changes in gene expression accompany this transition from pond to acute infection including, but not limited to, induction of vieA, which encodes a phosphodiesterase that hydrolyzes the second messenger cyclic di-GMP, and ToxR-regulated genes, including those for cholera toxin and the self-aggregative toxin-coregulated pilus (TCP). In addition several genes are repressed, such as those for the chitin-binding mannose-sensitive hemagglutinin pilus (MSHA) and the stress sigma factor RpoS. At a late stage of infection (bottom left panel), V. cholerae alters its gene expression again in order to detach from the epithelium - the 'escape response' - and to prepare for transmission to another host (for example, household spread) or entry into the aquatic environment. The late-stage changes include induction of genes for c-di-GMP synthesis (diguanylyl cyclases), nutrient-scavenging systems (such as the ferric uptake regulation (Fur) regulon) and motility (Fla) as well as repression of genes such as those for chemotaxis (Che) and the ToxR regulon. The resulting 'motile but non-chemotactic' state contributes to hyperinfectivity. If bacteria shed in stool are not ingested by another host in a short period of time, then one of two fates awaits them (top panel): establishment in the aquatic environment by finding suitable nutrient sources such as chitin, or decay into an 'active but non-culturable' state. Upon exposure to chitin, V. cholerae induces several genes involved in adherence to and catabolism of chitin (the $\mathrm{ChiS}$ regulon), as well as those involved in genetic competence (the TfoX regulon). Biofilm formation on surfaces is mediated by induction of the Vps regulon, which encodes extracellular polysaccharide. During transition to the active but non-culturable state, large changes in gene expression occur as $V$. cholerae attempts to adapt to nutrient-poor conditions. These include induction of phosphate and nitrogen starvation genes ( $p h o B$ and $g \ln B-1$, respectively) and repression of translation machinery genes.

outbreak begins when either the already infected index case migrates and contaminates a new area or $V$. cholerae consumed from a natural environmental reservoir creates a new index case in a resident population. In both scenarios, it is possible that the acceleration of the outbreak results from hyperinfectious $V$. cholerae spreading rapidly from person to person, with only a short period of time spent in the environment. Transmission models that allow only stochastic ingestion of $V$. cholerae from the environment do not predict the steep rises in case numbers that are observed at the onset of outbreaks around the world, including those that occur on a biannual basis in Bangladesh ${ }^{11}$. However, models that incorporate hyperinfectivity ${ }^{83}$ better reflect these steep rises.

Maintenance of transmission through aquatic reservoirs. Endemic cholera occurs in regions with natural aquatic reservoirs of toxigenic and non-toxigenic $V$. cholerae, where the bacteria can persist either in a free-living state or in association with phytoplankton $^{84-86}$, zooplantkon ${ }^{85-88}$ or biotic and abiotic detritus $^{89,90}$. These interactions can be both beneficial and antagonistic ${ }^{91,92}$. The associations are generally 


\section{Box 2 | The Bacteriophage Inquiry}

In 1915-1917, Twort ${ }^{114}$ and d'Hérelle ${ }^{115}$ independently discovered bacteriophages. d'Hérelle went on to determine that bacterial infection in animal models could be attenuated by bacteriophages, and in 1925 patients with bubonic plague improved after d'Hérelle injected bacteriophages directly into their buboes (swollen lymph nodes $)^{116}$. These discoveries led to the Bacteriophage Inquiry, which had a mandate to study cholera bacteriophage therapy in India ${ }^{116}$. Their first therapy trial compared 244 untreated patients with cholera with 219 patients with cholera who were treated with vibriophages; the untreated group had $20 \%$ mortality whereas the treated group had $6.8 \%\left(\chi^{2}, p<0.01\right)$. Other studies showed similar results ${ }^{5,116,117}$. Despite several limitations specific to the era, the Inquiry had achieved enough success to scale up their efforts ${ }^{5}$.

For the years of 1928, 1929 and 1934, totals of 36,000, 130,823 and 871,316 vibriophage doses were prepared and disseminated by the Inquiry staff at the time that outbreaks began in specific study communities in India ${ }^{116}$. Vibriophages were also disseminated into community drinking water sources for prophylaxis. The death rates from cholera were compared with control communities for before and after the interventions. The triennial death rates from cholera fell from 30 to 2 per 10,000 in regions that were treated with vibriophages ${ }^{117}$. Although these studies had multiple limitations, they do provide the only available data for the efficacy of vibriophage therapy. Advances in rehydration and antibiotic therapy in the 1930s and 1940s made bacteriophage trials based on mortality end points unethical and statistically challenging ${ }^{116,118}$.
$V$. cholerae may rapidly become $\mathrm{ABNC}$ when it leaves the human host and enters aquatic environments. Studies with patient- and in vitro-derived V. cholerae that were dialysed in pond water demonstrated declines of $60 \%$ and $90 \%$ in culturability at 5 hours and 24 hours, respectively ${ }^{65}$. Microarray analysis demonstrated that there were rapid and striking transcriptional changes as the bacteria entered the ABNC state ${ }^{65}$. These changes included the induction of genes for phosphate and fixed-nitrogen scavenging and the repression of protein synthesis and energy metabolism genes, consistent with the low levels of carbon sources, phosphate and fixed nitrogen that are often found in aquatic environments. It remains to be tested whether the inclusion of particulate matter, which was filtered out from the pond water in the above study, will prolong the culturability of $V$. cholerae. With such rapid declines in culturability being possible, investigators have asked whether the majority of $V$. cholerae cells infecting humans from the environment are ABNC or culturable cells. One hypothesis states that if culturable cells are more infectious than $\mathrm{ABNC}$ cells, then the $\mathrm{ID}_{50}$ calculated by total cell counts would increase as the percent of culturable cells decreases in a population. This was borne out in recent experiments, suggesting that the main contributors to human infection are probably culturable $V$. cholerae cells ${ }^{65}$. Future mathematical models for the transmission of $V$. cholerae should incorporate the possibility of a decay rate in culturability.

\section{V. cholerae lytic bacteriophage}

Biology of vibriophages. Eighty years ago, it was proposed that bacteriophages might control natural populations of pathogens ${ }^{99,100}$. More recent studies in marine microbiology have revealed the elegant balance between bacteriophages and their cyanobacterial prey ${ }^{101}$. From a clinical standpoint, bacteriophages could, in principle, be used for prophylaxis or to treat infections. Indeed, 'phage therapy' against cholera and other diseases has been documented (BOX 2). There are at least 200 bacteriophage species that infect $V$. cholerae, known as vibriophages ${ }^{102,103}$. The filamentous, lysogenic CTXФ vibriophage is one of the best characterized because it carries the genes that encode cholera toxin ${ }^{22}$. The first tailed vibriophage genome to be sequenced was that of $\kappa 139$; this vibriophage can be both lysogenic and lytic. The main mechanism by which $V$. cholerae $\mathrm{O} 1$ becomes resistant to $\kappa 139$ and many other vibriophages is mutation of the $r f b$ gene cluster that encodes the enzymes for the synthesis of LPS ${ }^{104}$. In the absence of the LPS O antigen receptor the vibriophage cannot bind or infect the bacterial cell. All $r f b$-null mutants of $V$. cholerae $\mathrm{O} 1$ that have been tested to date are attenuated ${ }^{65,67,105}$.

Vibriophages in the environment. It was discovered in the 1930s that cholera cases were positively correlated with the isolation of vibriophages in the aquatic environment ${ }^{106}$. In modern times, a delayed positive concordance between a rise in the number of cholera cases and a subsequent rise of vibriophages in the environment has been described ${ }^{107}$. A model developed by Jensen et al. predicts that if an outbreak is initiated by an increase in 


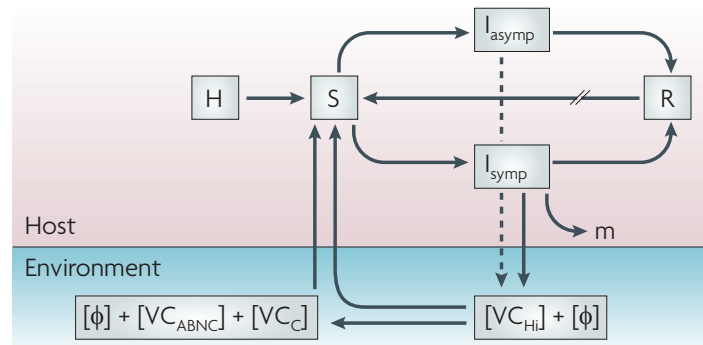

Figure 4 | A combined model for the transmission of cholera from the perspective of the host and microorganisms. The total population $(\mathrm{H})$ feeds the pool of susceptible hosts (S) that become infectious (I) after consuming Vibrio cholerae from an environmental source, with or without lytic bacteriophages $(\Phi)$. Infected individuals are symptomatic $\left(I_{\text {symp }}\right)$ or asymptomatic (I and recover $(\mathrm{R})$ through the actions of their immune systems and possibly those of lytic bacteriophages, or succumb to the infection $(\mathrm{m})$. Recovered individuals will re-enter the susceptible pool at varying rates (//) depending on the degree of protective immunity. Lytic phages and hyperinfectious $\mathrm{V}$. cholerae $\left(\mathrm{VC}_{\mathrm{Hi}}\right)$ are shed by the symptomatic host in varying concentrations; asymptomatic hosts shed far fewer bacteria (dashed line). $\mathrm{VC}_{\mathrm{Hi}}$ cells rapidly passage to the next host, persist in the environment as culturable cells with unknown infectivity $\left(\mathrm{VC}_{\mathrm{C}}\right)$ or decay into an 'active but non-culturable' state $\left(V_{\mathrm{ABNC}}\right)$ with reduced infectivity. All three cell types plus aggregate bacteria (not shown) probably have mixed roles as environmental reservoirs for future outbreaks.

$V$. cholerae in the environment, vibriophages will subsequently increase in density, ultimately promoting a decline in the outbreak ${ }^{108}$. At the International Centre for Diarrhoeal Disease Research, Bangladesh (ICDDRB), an increase in cholera cases was associated with a concordant but delayed rise in the proportion of cholera patients with vibriophages in their stools ${ }^{109}$. The vibriophages identified in this study were lytic vibriophages (JSF4), although multiple types of vibriophage can be isolated from patients ${ }^{107}$. The titres of vibriophages in rice water stool ranged from $10^{2}$ to $10^{8}$ plaque forming units per millilitre, which is consistent with other publications ${ }^{65,67}$. Despite the presence of the lytic vibriophages, the stools were all positive for $V$. cholerae, and $V$. cholerae typically outnumbered vibriophages by at least one order of magnitude. Subsequent studies have shown that most bacterial isolates from the stools were still susceptible to the vibriophages ${ }^{65,67}$. It is not yet clear why vibriophages in the human intestine fail to clear V. cholerae infection, but this 'failure' may play an important part in propagating the clonal expansion of phages during outbreaks ${ }^{109}$.

Transmission from the perspective of the vibriophage. Modern experiments have begun to test the hypothesis that phages can attenuate bacterial transmission. For example, motility assays using dark-field microscopy were used to rapidly screen rice water stools for vibriophages; stools that were dark-field negative (containing no motile vibrios) were more likely to contain lytic vibriophages and to have low culturable-cell counts but similar total cell counts ${ }^{77}$. By this indirect measure for phages, it was found that at least half of the patients with cholera that were seen over a 5 -year period at the ICDDRB harboured vibriophages, and household contacts of a vibriophage-positive index case were less likely to be infected with $V$. cholerae than individuals who were in contact with a patient who was not phage positive ${ }^{77}$. These results are consistent with the finding that there is a tenfold drop in the infectious dose of V. cholerae when the stool of the host harbours vibriophages ${ }^{67}$.

$V$. cholerae typically outnumbers lytic bacteriophages immediately after passage from the host ${ }^{65,67}$. In laboratory experiments using human-shed rice water stools, bacteriophages have an initial burst in replication during the first few hours in the aquatic environment and can reach a bacteriophage to bacterium ratio of approximately 1 to 1 by 24 hours $^{65,67}$. Although hyperinfectivity can be maintained for several hours after passage from the host ${ }^{65,70}$, the loss of culturability (discussed above) and the bloom of bacteriophages in the aquatic environment probably combine to block transmission ${ }^{65}$. Control experiments with in vitro-derived $V$. cholerae and bacteriophages confirm that bacteriophages can limit the burden of infection $^{65,67}$. However, bacteriophages are never able to block infection completely, because mutants of $V$. cholerae that lack mature LPS escape predation and colonize mice, albeit at reduced numbers. As mentioned, LPS mutants are attenuated and may be lost in the natural life cycle of $V$. cholerae $e^{65,67,105}$.

In summary, the dynamic interaction between bacteriophage and bacterium in pond water suggests that a model of cholera transmission should incorporate a measure for the rapid decay of bacterial culturability and the predation by bacteriophages. In a closed experimental system, transmission of $V$. cholerae may be minimized when these two factors combine in the aquatic environment. Therefore, there is likely to be a fitness advantage for $V$. cholerae that makes a rapid transfer to the next host, when culturability is still high and the concentration of bacteriophages is still low. In this Review, several models have been referenced that offer different explanations for the rise and fall of outbreaks, from the perspective of the host (clinical spectrum and herd immunity), the bacterium (hyperinfectivity) and the bacteriophage (predation). We have incorporated several of the published models into one working diagram to provoke thought on how these factors may interact in the natural environment (FIG. 4). Having a better sense of how the integrated model functions may reveal opportunities for public health interventions.

\section{Concluding remarks}

A transmission model that accurately predicts the magnitude of an emerging outbreak would provide public health authorities with useful information to appropriately scale their responses. Interventions that target vital steps in transmission might be effective for the prevention of outbreaks. Host immunity, pathogen hyperinfectivity and phages are all factors that can be leveraged for outbreak 
control. For example, centralized waste management centres often fail in resource-poor settings. As symptomatic patients shed more $V$. cholerae, and the $V$. cholerae in rice water stool is hyperinfectious, cholera outbreaks may best be stopped at the source, by reducing human exposure to freshly passed, hyperinfectious stool. In other words, while maintaining centralized management, decentralized efforts for directed waste management at the unit of the household should be encouraged and tested. This concept highlights the crucial importance of already proven but simple household-based interventions, such as the use of narrow-mouthed jugs, chlorination of stored water and hand washing, for disease prevention ${ }^{110,111}$.
Much remains to be learned about vaccine efficacy in the natural setting of cholera. Breaking from traditional measures of vaccine efficacy by incorporating the benefits of herd immunity will continue to unveil the true impact of both existing vaccines and vaccines in development. Furthermore, understanding the impact of bacteriophage predation and how vaccines lower the susceptible pool to the point that transmission cannot be perpetuated are important areas for future research. Answers to the many questions posed in this Review are crucial for resource-poor countries, like Zimbabwe, to optimize the use of a limited vaccine supply and to combat the ill effects of failed sanitation.
1. Bryce, J., Boschi-Pinto, C., Shibuya, K. \& Black, R. E. WHO estimates of the causes of death in children. Lancet 365, 1147-1152 (2005)

2. Kosek, M., Bern, C. \& Guerrant, R. L. The global burden of diarrhoeal disease, as estimated from studies published between 1992-2000 Bull. World Health Organ. 81, 197-204 (2003).

3. Sack, D. A., Sack, R. B. \& Chaignat, C. L. Getting serious about cholera. N. Engl. J. Med. 355, 649-651 (2006).

4. Pollitzer, R., Swaroop, S. \& Burrows, W. Cholera. Monogr. Ser. World Health Organ. 58, 1001-1019 (1959).

5. Pasricha, C. L., de Monte, A. J. H. \& O’Flynn, E. G. Bacteriophage in the treatment of cholera. Ind. Med. Gaz. 71, 61-68 (1936).

6. Asheshov, I. \& Lahiri, M. N. The treatment of cholera with bacteriophage. Ind. Med. Gaz. 179-184 (1931).

Wachsmuth, I. K., Blake, P. A. \& Olsvik, $\varnothing$ (eds) in Vibrio cholerae and Cholera: Molecular to Global Perspectives. (ASM, Washington DC,1994).

8. WHO. Cholera 2007. Wkly Epidemiol. Rec. 83, 261-284 (2008).

9. Koch, R. An address on cholera and its bacillus. BMJ 2, 403-407 (1884)

10. Pacini, F. Osservazioni microscopiche e deduzioni patalogiche sul cholera asiatico. Gazzetta Medica Italiana Federativa Toscana, Firenze 4 (1854) (in Italian)

11. Longini, I. M. Jr et al. Epidemic and endemic cholera trends over a 33-year period in Bangladesh. J. Infect. Dis. 186, 246-251 (2002).

12. Udden, S. M. et al. Acquisition of classical CTX prophage from Vibrio cholerae O141 by El Tor strains aided by lytic phages and chitin-induced competence. Proc. Natl Acad. Sci. USA 105, 11951-11956 (2008)

13. Faruque, S. M. et al. Genomic analysis of the Mozambique strain of Vibrio cholerae $\mathrm{O} 1$ reveals the origin of El Tor strains carrying classical CTX prophage. Proc. Natl Acad. Sci. USA 104, 5151-5156 (2007)

14. Nair, G. B. et al. New variants of Vibrio cholerae 01 biotype El Tor with attributes of the classical biotype from hospitalized patients with acute diarrhoea in Bangladesh. J. Clin. Microbiol. 40, 3296-3299 (2002).

15. Waldor, M. K., Colwell, R. \& Mekalanos, J. J. The Vibrio cholerae $\mathrm{O} 139$ serogroup antigen includes an O-antigen capsule and lipopolysaccharide virulence determinants. Proc. Natl Acad. Sci. USA 91 , 11388-11392 (1994).

16. Sack, R. B. et al. A 4-year study of the epidemiology of Vibrio cholerae in four rural areas of Bangladesh. J. Infect. Dis. 187, 96-101 (2003).

17. Sharma, N. C., Mandal, P. K., Dhillon, R. \& Jain, M. Changing profile of Vibrio cholerae O1, O139 in Delhi $\mathcal{L}$ its periphery (2003-2005). Indian J. Med. Res. 125, 633-640 (2007)

18. Holmgren, J. Actions of cholera toxin and the prevention and treatment of cholera. Nature 292 , 413-417 (1981)

19. Taylor, R. K., Miller, V. L., Furlong, D. B. \& Mekalanos, J. J. Use of $p h o A$ gene fusions to identify a pilus colonization factor coordinately regulated with cholera toxin. Proc. Natl Acad. Sci. USA 84, 2833-2837 (1987).

20. Herrington, D. A. et al. Toxin, toxin-coregulated pili, and the toxR regulon are essential for Vibrio cholerae pathogenesis in humans. J. Exp. Med. 168, 1487-1492 (1988).
This article confirms the importance in human volunteers of virulence factors that were originally identified using animal models of infection.

21. Kirn, T. J., Lafferty, M. J., Sandoe, C. M. \& Taylor, R. K. Delineation of pilin domains required for bacterial association into microcolonies and intestinal colonization by Vibrio cholerae. Mol. Microbiol. 35, 896-910 (2000).

22. Waldor, M. K. \& Mekalanos, J. J. Lysogenic conversion by a filamentous phage encoding cholera toxin. Science 272, 1910-1914 (1996)

This paper reports the discovery that the genes for cholera toxin are encoded on a lysogenic bacteriophage.

23. Burrus, V., Marrero, J. \& Waldor, M. K. The current ICE age: biology and evolution of SXT-related integrating conjugative elements. Plasmid 55, 173-183 (2006).

24. Oliver, J. D. The viable but nonculturable state in bacteria. J. Microbiol. 43, 93-100 (2005).

25. Vezzulli, L., Guzman, C. A., Colwell, R. R. \& Pruzzo, C. Dual role colonization factors connecting Vibrio cholerae's lifestyles in human and aquatic environments open new perspectives for combating infectious diseases. Curr. Opin. Biotechnol. 19, 254-259 (2008).

26. Lipp, E. K., Huq, A. \& Colwell, R. R. Effects of global climate on infectious disease: the cholera model. Clin. Microbiol. Rev. 15, 757-770 (2002).

27. Phillips, R. A. Water and electrolyte losses in cholera. Fed. Proc. 23, 705-712 (1964).

28. Cash, R. A. et al. Response of man to infection with Vibrio cholerae. I. Clinical, serologic, and bacteriologic responses to a known inoculum. J. Infect. Dis. 129 , 45-52 (1974).

29. Cash, R. A. et al. Response of man to infection with Vibrio cholerae. II. Protection from illness afforded by previous disease and vaccine. J. Infect. Dis. 130 325-333 (1974)

30. Feachem, R. G. Environmental aspects of cholera epidemiology. III. Transmission and control. Trop. Dis Bull. 79, 1-47 (1982)

31. Kaper, J. B., Morris, J. G. Jr \& Levine, M. M. Cholera Clin. Microbiol. Rev. 8, 48-86 (1995).

32. Mosley, W. H., Ahmad, S., Benenson, A. S. \& Ahmed, A. The relationship of vibriocidal antibody titre to susceptibility to cholera in family contacts of cholera patients. Bull. World Health Organ. 38, 777-785 (1968).

33. Glass, R. I. et al. Endemic cholera in rural Bangladesh, 1966-1980. Am. J. Epidemiol. 116, 959-970 (1982).

34. Deen, J. L. et al. The high burden of cholera in children comparison of incidence from endemic areas in Asia and Africa. PLoS Negl. Trop. Dis. 2, e 173 (2008).

35. Harris, J. B. et al. Susceptibility to Vibrio cholerae infection in a cohort of household contacts of patients with cholera in Bangladesh. PLoS Negl. Trop. Dis. 2, e221 (2008)

36. Glass, R. I. ¿ Black, R. E. in Cholera (eds Barua, D. \& Greenough, W. B.) 129-154 (Plenum Medical Book Co., New York, 1992).

37. Holmberg, S. D. et al. Foodborne transmission of cholera in micronesia. Lancet 1, 325-328 (1984).

38. McCormack, W. M., Islam, M. S., Fahimuddin, M. \& Mosley, W. H. A community study of inapparent cholera infections. Am. J. Epidemiol. 89, 658-664 (1969).
39. Woodward, W. E. \& Mosley, W. H. The spectrum of cholera in rural Bangladesh. II. Comparison of El Tor Ogawa and classical Inaba infection. Am. J. Epidemiol. 96, 342-351 (1972).

40. Bart, K. J., Huq, Z., Khan, M. \& Mosley, W. H. Seroepidemiologic studies during a simultaneous epidemic of infection with El Tor Ogawa and classical Inaba Vibrio cholerae. J. Infect. Dis. 121 Suppl. 121:17 + (1970).

41. Glass, R. I. et al. Predisposition for cholera of individuals with $\mathrm{O}$ blood group. Possible evolutionary significance. Am. J. Epidemiol. 121, 791-796 (1985).

42. Tacket, C. O. et al. Extension of the volunteer challenge model to study South American cholera in a population of volunteers predominantly with blood group antigen O. Trans. R. Soc. Trop. Med. Hyg. 89 , 75-77 (1995).

43. Swerdlow, D. L. et al. Severe life-threatening cholera associated with blood group $\mathrm{O}$ in Peru: implications for the Latin American epidemic. J. Infect. Dis. 170 468-472 (1994)

44. Roy, S. K. et al. Zinc supplementation in children with cholera in Bangladesh: randomised controlled trial. BMJ 336, 266-268 (2008).

45. Larocque, R. C. et al. A variant in long palate, lung and nasal epithelium clone 1 is associated with cholera in a Bangladeshi population. Genes Immun. 10, 267-272 (2009).

46. Flach, C. F. et al. Broad up-regulation of innate defence factors during acute cholera. Infect. Immun. $\mathbf{7 5}$, 2343-2350 (2007)

47. Rothbaum, R. J., Maur, P. R. \& Farrell, M. K. Serum alkaline phosphatase and zinc undernutrition in infants with chronic diarrhoea. Am. J. Clin. Nutr. 35, 595-598 (1982).

48. Levine, M. M. et al. Immunity of cholera in man relative role of antibacterial versus antitoxic immunity. Trans. R. Soc. Trop. Med. Hyg. 73, 3-9 (1979).

49. Clemens, J. D. et al. Biotype as determinant of natural immunising effect of cholera. Lancet 337, 883-884 (1991).

50. Clemens, J. D. et al. Field trial of oral cholera vaccines in Bangladesh: results from three-year follow-up. Lancet 335, 270-273 (1990).

This trial shows that immunity from killed whole-cel vaccine is of limited duration.

51. Clemens, J. D. et al. Field trial of oral cholera vaccines in Bangladesh. Lancet 2, 124-127 (1986). This article describes the largest field trial of a cholera vaccine to date, showing high efficacy over a short period.

52. Lucas, M. E. et al. Effectiveness of mass oral cholera vaccination in Beira, Mozambique. N. Engl. J. Med. 352, 757-767 (2005)

53. Qadri, F. et al. Peru-15, a live attenuated oral cholera vaccine, is safe and immunogenic in Bangladeshi toddlers and infants. Vaccine 25, 231-238 (2007).

54. Schild, S., Nelson, E. J. \& Camilli, A. Immunization with Vibrio cholerae outer membrane vesicles induces protective immunity in mice. Infect. Immun. 76, 4554-4563 (2008).

55. Rhie, G. E., Jung, H. M., Park, J., Kim, B. S. \& Mekalanos, J. J. Construction of cholera toxin B subunit-producing Vibrio cholerae strains using the Mariner-FRT transposon delivery system. FEMS Immunol. Med. Microbiol. 52, 23-28 (2008).

56. Longini, I. M. Jr, Helloran, M. E. \& Nizam, A. Modelbased estimation of vaccine effects from community vaccine trials. Stat. Med. 21, 481-495 (2002). 
57. Ali, M. et al. Herd immunity conferred by killed oral cholera vaccines in Bangladesh: a reanalysis. Lance 366, 44-49 (2005)

This study shows that killed whole-cell vaccines provide protection to non-vaccinated individuals when a large enough percentage of the population is vaccinated.

58. Longini, I. M. Jr et al. Controlling endemic cholera with oral vaccines. PLoS Med. 4, e336 (2007).

59. Koelle, K., Rodo, X., Pascual, M., Yunus, M. \& Mostafa, G. Refractory periods and climate forcing in cholera dynamics. Nature 436, 696-700 (2005).

60. Koelle, K., Pascual, M. \& Yunus, M. Pathogen adaptation to seasonal forcing and climate change. Proc. Biol. Sci. 272, 971-977 (2005).

61. Koelle, K. \& Pascual, M. Disentangling extrinsic from intrinsic factors in disease dynamics: a nonlinear time series approach with an application to cholera. $\mathrm{Am}$. Nat. 163, 901-913 (2004).

62. King, A. A., Ionides, E. L., Pascual, M. \& Bouma, M. J. Inapparent infections and cholera dynamics. Nature 454, 877-880 (2008).

63. Schild, S., Bishop, A. L. \& Camilli, A. Ins and outs of Vibrio cholerae. ASM Microbe Magazine 3, 131-136 (2008).

64. Wiles, S., Hanage, W. P., Frankel, G. \& Robertson, B. Modelling infectious disease - time to think outside the box? Nature Rev. Microbiol. 4, 307-312 (2006).

65. Nelson, E. J. et al. Transmission of Vibrio cholerae is antagonized by lytic phage and entry into the aquatic environment. PLoS Pathog. 4, e 1000187 (2008). The work described in this article shows that the rapid loss of culturability of human-shed $V$. cholera and the rise of lytic bacteriophages in pond water combine to limit transmission.

66. Butler, S. M. et al. Cholera stool bacteria repress chemotaxis to increase infectivity. Mol. Microbiol. 60 417-426 (2006)

67. Zahid, M. S. et al. Effect of phage on the infectivity of Vibrio cholerae and emergence of genetic variants. Infect. Immun. 76, 5266-5273 (2008)

68. Wiles, S., Dougan, G. \& Frankel, G. Emergence of a 'hyperinfectious' bacterial state after passage of Citrobacter rodentium through the host gastrointestinal tract. Cell. Microbiol. 7, 1163-1172 (2005).

69. Alam, A. et al. Hyperinfectivity of human-passaged Vibrio cholerae can be modeled by growth in the infant mouse. Infect. Immun. 73, 6674-6679 (2005).

70. Merrell, D. S. et al. Host-induced epidemic spread of the cholera bacterium. Nature 417, 642-645 (2002). This paper reports that $V$. cholerae shed in huma rice water stool is transiently hyperinfectious.

71. Larocque, R. C. et al. Transcriptional profiling of Vibrio cholerae recovered directly from patient specimens during early and late stages of human infection. Infect Immun. 73, 4488-4493 (2005).

72. Butler, S. M. \& Camilli, A. Both chemotaxis and net motility greatly influence the infectivity of Vibrio cholerae. Proc. Natl Acad. Sci. USA 101, 5018-5023 (2004).

73. Butler, S. M. \& Camilli, A. Going against the grain: chemotaxis and infection in Vibrio cholerae. Nature Rev. Microbiol. 3, 611-620 (2005).

74. Nielsen, A. T. et al. RpoS controls the Vibrio cholerae mucosal escape response. PLoS Pathog. 2, e109 (2006).

This manuscript describes a $V$. cholerae genetic programme that promotes the release of bacteria from the intestinal epithelium for their subsequent expulsion in stool.

75. Schild, S. et al. Genes induced late in infection increase fitness of Vibrio cholerae after release into the environment. Cell Host Microbe 2, 264-277 (2007). This investigation identifies $V$. cholerae genes that are induced late during infection and provide a fitness advantage in aquatic environments.

76. Morris, J. G. Jr. et al. Vibrio cholerae $\mathrm{O} 1$ can assume a chlorine-resistant rugose survival form that is virulent for humans. J. Infect. Dis. 174, 1364-1368 (1996).

77. Nelson, E. J. et al. Complexity of rice-water stool from patients with Vibrio cholerae plays a role in the transmission of infectious diarrhoea. Proc. Natl Acad. Sci. USA 104, 19091-19096 (2007).

78. Faruque, S. M. et al. Transmissibility of cholera: in vivoformed biofilms and their relationship to infectivity and persistence in the environment. Proc. Natl Acad. Sci. USA 103, 6350-6355 (2006)

79. Levine, M. M. et al. in Acute Enteric Infections in Children. New Prospects for Treatment and Prevention. (eds Holme, T., Holmgren, J., Merson, M. H. \& Möllby, R.)
443-459 (Elsevier/North-Holland Biomedical Press, Amsterdam, 1981)

80. Brayton, P. R., Tamplin, M. L., Huq, A. \& Colwell, R. R. Enumeration of Vibrio cholerae $\mathrm{O} 1$ in Bangladesh waters by fluorescent-antibody direct viable count. Appl. Environ. Microbiol 53, 2862-2865 (1987)

81. Merrell, D. S., Hava, D. L. \& Camilli, A. Identification of novel factors involved in colonization and acid tolerance of Vibrio cholerae. Mol. Microbiol. 43, 1471-1491 (2002).

82. Merrell, D. S. \& Camilli, A. The cadA gene of Vibrio cholerae is induced during infection and plays a role in acid tolerance. Mol. Microbiol. 34, 836-849 (1999).

83. Hartley, D. M., Morris, J. G. Jr \& Smith, D. L. Hyperinfectivity: a critical element in the ability of V. cholerae to cause epidemics? PLoS Med. 3, e7 (2006)

This study shows that factoring hyperinfectivity into a standard mathematical model for transmission yields a model that is more representative of the case loads that are observed in the field.

84. Tamplin, M. L., Gauzens, A. L., Huq, A., Sack, D. A. \& Colwell, R. R. Attachment of Vibrio cholerae serogroup O1 to zooplankton and phytoplankton of Bangladesh waters. Appl. Environ. Microbiol. 56, 1977-1980 (1990)

85. Shukla, B. N., Singh, D. V. \& Sanyal, S. C. Attachment of non-culturable toxigenic Vibrio cholerae $\mathrm{O} 1$ and non-O1 and Aeromonas spp. to the aquatic arthropod Gerris spinolae and plants in the River Ganga, Varanasi. FEMS Immunol. Med. Microbiol. 12 113-120 (1995).

86. Halpern, M., Broza, Y. B., Mittler, S., Arakawa, E. \& Broza, M. Chironomid egg masses as a natural reservoir of Vibrio cholerae non-O1 and non-O139 in freshwater habitats. Microb. Ecol. 47, 341-349 (2004)

87. Huq, A. et al. Ecological relationships between Vibrio cholerae and planktonic crustacean copepods. Appl. Environ. Microbiol. 45, 275-283 (1983)

88. Abd, H., Weintraub, A. \& Sandstrom, G. Intracellular survival and replication of Vibrio cholerae O139 in aquatic free-living amoebae. Environ. Microbiol. 7 1003-1008 (2005)

89. Islam, M. S. et al. Biofilm acts as a microenvironment for plankton-associated Vibrio cholerae in the aquatic environment of Bangladesh. Microbiol. Immunol. 51, 369-379 (2007).

90. Alam, M. et al. Viable but nonculturable Vibrio cholerae $\mathrm{O} 1$ in biofilms in the aquatic environment and their role in cholera transmission. Proc. Natl Acad. Sci. USA 104, 17801-17806 (2007).

91. Long, R. A. et al. Antagonistic interactions among marine bacteria impede the proliferation of Vibrio cholerae. Appl. Environ. Microbiol. 71, 8531-8536 (2005)

92. Matz, C. et al. Biofilm formation and phenotypic variation enhance predation-driven persistence of Vibrio cholerae. Proc. Natl Acad. Sci. USA 102 16819-16824 (2005)

93. Nalin, D. R., Daya, V., Reid, A., Levine, M. M. \& Cisneros, L. Adsorption and growth of Vibrio cholerae on chitin. Infect. Immun. 25, 768-770 (1979)

94. Meibom, K. L. et al. The Vibrio cholerae chitin utilization program. Proc. Natl Acad. Sci. USA 101 2524-2529 (2004)

95. Bartlett, D. H. \& Azam, F. Microbiology. Chitin, cholera and competence. Science 310, 1775-1777 (2005).

96. Stine, O. C. et al. Seasonal cholera from multiple small outbreaks, rural Bangladesh. Emerg. Infect. Dis. 14, 831-833 (2008).

97. Colwell, R. R. et al. Viable but non-culturable Vibrio cholerae and related pathogens in the environment: implications for release of genetically engineered microorganisms. Nature Biotech. 3, 817-820 (1985)

This article describes the phenomenon of entry of bacterial pathogens into a metabolically active but non-culturable state on incubation in aquatic environments.

98. Kell, D. B., Kaprelyants, A. S., Weichart, D. H. Harwood, C. R. \& Barer, M. R. Viability and activity in readily culturable bacteria: a review and discussion of the practical issues. Antonie Van Leeuwenhoek 73 169-187 (1998)

99. d'Herelle, F. The Bacteriophage and its Behavior (Bailliere, Tindall \& Cox, London, 1926)

100. Morison, J., Rice, E. M. \& Choudhury, B. K. P. Bacteriophage in the treatment and prevention of cholera. Indian J. Med. Res. 790-857 (1933).
101. Suttle, C. A. Viruses in the sea. Nature 437, 356-361 (2005)

102. Kapfhammer, D., Blass, J., Evers, S. \& Reidl, J. Vibrio cholerae phage K139: complete genome sequence and comparative genomics of related phages. J. Bacteriol. 184, 6592-6601 (2002).

103 Glass, R. I., Lee, J. V., Huq, M. I., Hossain, K. M. \& Khan, M. R. Phage types of Vibrio cholerae $\mathrm{O} 1$ biotype El Tor isolated from patients and family contacts in Bangladesh: epidemiologic implications. J. Infect. Dis. 148, 998-1004 (1983).

104. Nesper, J. et al. Comparative and genetic analyses of the putative Vibrio cholerae lipopolysaccharide core oligosaccharide biosynthesis (wav) gene cluster. Infect. Immun. 70, 2419-2433 (2002).

105. Nesper, J. et al. Role of Vibrio cholerae 0139 surface polysaccharides in intestinal colonization. Infect. Immun. 70, 5990-5996 (2002).

106. Pasricha, C. L., Monte, A. J. \& Gupta, S. K. Season variations of cholera bacteriophage in natural waters and in man in Calcutta during the year 1930. Ind Med. Gaz. 66, 543-550 (1931).

107. Faruque, S. M. et al. Seasonal epidemics of cholera inversely correlate with the prevalence of environmental cholera phages. Proc. Natl Acad. Sci. USA 102, 1702-1707 (2005).

108. Jensen, M. A., Faruque, S. M., Mekalanos, J. J. \& Levin, B. R. Modeling the role of bacteriophage in the control of cholera outbreaks. Proc. Natl Acad. Sci. USA 103, 4652-4657 (2006).

109. Faruque, S. M. et al. Self-limiting nature of seasonal cholera epidemics: role of host-mediated amplification of phage. Proc. Natl Acad. Sci. USA 102, 6119-6124 (2005).

This paper proposes that lytic bacteriophages increase in density in the environment to limit the duration of cholera outbreaks.

110. Deb, B. C. et al. Studies on interventions to prevent El Tor cholera transmission in urban slums. Bull. World Health Organ. 64, 127-131 (1986).

111. St. Louis, M. E. et al. Epidemic cholera in West Africa: the role of food handling and high-risk foods. Am. J. Epidemiol. 131, 719-728 (1990)

112. Bhuiyan, T. R. et al. Cholera caused by Vibrio cholerae $\mathrm{O} 1$ induces T-cell responses in the circulation. Infect. Immun. 77, 1888-1893 (2009).

113. Harris, A. M. et al. Antigen specific memory B-cell responses to Vibrio cholerae $\mathrm{O} 1$ infection in Bangladesh. Infect. Immun. 15 Jun 2009 (doi: 10.1128/IAI.00369-09)

114. Twort, F. W. Investigations on the nature of ultramicroscopic viruses. Lancet 2, 1241-1243 (1915).

115. d'Hêrelle, F. Sur un microorganism antagoniste des bacilles dysenteriques. C. R. Acad. Sci. 165, 373-375 (1917) (in French)

116. Summers, W. C. Cholera and plague in India: the bacteriophage inquiry of 1927-1936. J. Hist. Med Allied Sci. 48, 275-301 (1993).

117. Morison, J., Rice, E. \& Pal Choudhury, B. Bacteriophage in the treatment and prevention of cholera. Indian J. Med. Res. 21, 790-907 (1934).

118. Marcuk, L. M. et al. Clinical studies of the use of bacteriophage in the treatment of cholera. Bull. World Health Organ. 45, 77-83 (1971).

Acknowledgements

A.C. is supported by the National Institutes of Health grants R01 Al045746 and R01 Al055058

\section{DATABASES}

CDC Diseases and Conditions: http://www.cdc.gov/

DiseasesConditions

cholera

Entrez Gene: http://www.ncbi.nlm.nih.gov/entrez/query.

fcgi?db=gene

gln $B-1 \mid$ phoB $\mid$ vieA

Entrez Genome Project: http://www.ncbi.nlm.nih.gov/ entrez/query.fcgi?db=geneomepri Escherichia coli K12 | Helicobacter pylori |Vibrio cholerae UniProtKB: http://www.uniprot.ors GbpA|LPLUNC1 $\mid$ RpoS $\mid$ ToxR

FURTHER INFORMATION

Andrew Camilli's homepage: http://www.tufts.edu/sackler/ microbiology/faculty/camilli/index.html ALL LINKS ARE ACTIVE IN THE ONLINE PDF 